\title{
A Necessary Condition for Optimal Control of Forward-Backward Stochastic Control System with Lévy Process in Nonconvex Control Domain Case
}

\author{
Hong Huang $\mathbb{D}^{1}{ }^{1}$ Xiangrong Wang $\mathbb{D}^{2}{ }^{2}$ and Ying $\mathrm{Li} \mathbb{i D}^{3,4}$ \\ ${ }^{1}$ School of Data and Computer Science, Shandong Women's University, Jinan 250300, China \\ ${ }^{2}$ Institute of Financial Engineering, Shandong University of Science and Technology, Qingdao 266590, China \\ ${ }^{3}$ Office of Academic Research, Shandong Women's University, Jinan 250300, China \\ ${ }^{4}$ College of Computer Science and Engineering, Shandong University of Science and Technology, Qingdao 266590, China
}

Correspondence should be addressed to Ying Li; cherry_jn@126.com

Received 10 April 2020; Accepted 18 May 2020; Published 3 June 2020

Guest Editor: Yi Qi

Copyright (C) 2020 Hong Huang et al. This is an open access article distributed under the Creative Commons Attribution License, which permits unrestricted use, distribution, and reproduction in any medium, provided the original work is properly cited.

This paper analyzes one kind of optimal control problem which is described by forward-backward stochastic differential equations with Lévy process (FBSDEL). We derive a necessary condition for the existence of the optimal control by means of spike variational technique, while the control domain is not necessarily convex. Simultaneously, we also get the maximum principle for this control system when there are some initial and terminal state constraints. Finally, a financial example is discussed to illustrate the application of our result.

\section{Introduction}

Stochastic optimal control is an important matter that cannot be neglected in modern control theory in long days. As is known to all, Pontryagin's [1] maximum principle is one of the main ways to settle the stochastic optimal control problem. By introducing the Hamiltonian function, a necessary condition for the optimal control of stochastic control systems was given by him, which was called the maximum condition. From that time, plenty of works on this issue have been done. Peng [2] was the first one to prove the general maximum principle of the forward-backward stochastic control system with diffusion coefficient containing the control variable by the technique of the second-order Taylor expansion and the second-order duality. $\mathrm{He}$ [3] was also the first one to demonstrate the maximum principle of forward-backward stochastic control systems from the view of backward stochastic differential equations (BSDE). In Peng's paper [3], the control domain was convex (in local form); Xu [4] extended this conclusion to the case of the nonconvex control domain (in global form), but the control variables were not included in the diffusion coefficient. And these results were extended to the fully coupled case in the form of local and global by Shi and $\mathrm{Wu}$
[5, 6] in 1998 and in 2006, respectively. On the basis of these works, Situ [7] was the first to obtain the maximum principle of the forward stochastic control system with global form of random jumps in 1991. Shi and Wu [8] and Shi [9] acquired the maximum principle for a kind of forward-backward stochastic control system with Poisson jumps in the form of local and global, respectively. The fully coupled forward-backward stochastic control system was extended by Liu et al. [10] at the base of Shi and $\mathrm{Wu}$ [8], and in the meanwhile, they also obtained the maximum principle with the control system be constrained about initial-terminal state constraints. Considering that in real life, the decision makers could only get partial information but not complete information in most cases; many scholars have paid attention to the partial observable stochastic optimal control problem and have achieved many results (see, for example, [11-13]). Traditionally, when using a stochastic partial differential equation called the Zakai equation to transform a full-information optimal control problem to the partially observable case, scholars will encounter a difficult problem: an infinite-dimensional optimal control problem. Wang and $\mathrm{Wu}$ [14] proposed a backward separation approach and replaced the original state and observation equation with the Zakai equation, 
and lots of complicated stochastic calculi in infinite-dimensional spaces were avoided in this way. Based on this approach, Xiao [15] studied a partially observed optimal control of forwardbackward stochastic systems with random jumps and obtained the maximum principle and sufficient conditions of an optimal control under some certain convexity assumptions. Wang et al. [16] proved the maximum principles for forward-backward stochastic control systems with correlated state and observation noises. More recent conclusions of the partially observed stochastic control problem can be seen from the studies conducted by Wang et al. [17], Zhang et al. [18], and Xiong et al. [19].

In these years, through the study of mathematical economics and mathematical finance, many scholars turn their attention to the stochastic control system driven by Lévy process. In 2000, Nualart and Schoutens [20] built a pair of pairwise strongly orthonormal martingales which was called the Teugels martingale. Meanwhile, under some exponential moment conditions, they also obtained a martingale representation in that paper. Under these two important conclusions, for BSDE driven by the Teugels martingale, they [21] proved the existence and uniqueness theorem of its solution in the next year. From then on, a number of important results were proved: Meng and Tang [22] obtained the maximum principle of the forward stochastic control system driven by Lévy process. A necessary and sufficient condition for the existence of the optimal control of backward stochastic control systems driven by Lévy process was deduced by Tang and Zhang [23] through convex variation methods and duality techniques. For the forward-backward stochastic control system driven by Lévy process, there are also a lot of achievements: based on the existence and uniqueness theorem of FBSDEL [24], Zhang et al. [25] obtained a necessary condition of the optimal control and verification theorem, but in their control system, the backward state variables $y_{t}$ and $z_{t}$ did not enter the forward part. Wang and Huang [26] extended this result to the fully coupled control system and obtained the continuity result depending on parameters about FBSDEL and the local form maximum principle. Subsequently, Huang et al. [27] studied this control system with terminal state constraints and obtained the corresponding necessary maximum principle using Ekeland's variational. For more recent conclusions about the stochastic control problem driven by Lévy process, please refer to [28-30].

In this paper, we will study the optimal control problem for forward-backward stochastic control systems driven by Lévy process, which could be considered as a nonconvex control domain case that is extended from the result of [25].
With the technique of spike variation and Ekeland's variational principle, the maximum principle of this type of control system and the control system with initial and final state constraints are obtained.

The structure of this paper is as follows. Section 2 describes some of the preparations used in this paper. The maximum principle and the one with initial and terminal state constraints as the major results of this paper will be shown in Sections 3 and 4. As an application of the maximum principle, Section 5 gives an optimal consumption problem in the financial market. Section 6 is the summary of this article.

\section{Preliminary Statement}

Let $\left(\Omega, \mathscr{F}_{t}, P\right)$ be a complete probability space which satisfied the usual conditions, and the information structure is given by $\mathscr{F}_{t}$ which is generated by two processes: a standard Brownian motion $\left\{B_{t}\right\}_{0 \leq t \leq T}$ valued in $R^{d}$ and an independent 1-dimensional Lévy process $\left\{L_{t}\right\}_{0 \leq t \leq T}$ of the form $L_{t}=b_{t}+l_{t}$; here, $l_{t}$ is a pure jump process. And assume that Lévy measure $v$ satisfies the following two conditions; thereby, Lévy process $\left\{L_{t}\right\}_{0 \leq t \leq T}$ has moments in all orders.

(i) $\int_{R}\left(1 \wedge x^{2}\right) \nu(\mathrm{d} x)<\infty$.

(ii) $\int_{(-\varepsilon, \varepsilon)} e^{\lambda|x|} \nu(\mathrm{d} x)<\infty, \forall \varepsilon>0$ and some $\lambda>0$.

Denote $L_{t}^{1}=L_{t}, \triangle L_{t}=L_{t}-L_{t-}$, and $L_{t}^{i}=\sum_{0<s \leq t}\left(\triangle L_{s}\right)^{i}$ for $i \geq 2$. And let $Y_{t}^{i}=L_{t}^{i}-E\left[L_{t}^{i}\right](i \geq 1)$ be the compensated power jump process of order $i$; then, Teugels martingale is defined by $H_{t}^{i}=\sum_{j=1}^{i} c_{i j} Y_{t}^{j}$; the coefficients $c_{i j}$ correspond to orthonormalization of the polynomials $1, x, x^{2}, \ldots$ with respect to the measure $\mu(\mathrm{d} x)=v(\mathrm{~d} x)+\sigma^{2} \delta_{0}(\mathrm{~d} x)$.

Then, $\left\{H_{t}^{i}\right\}_{i=1}^{\infty}$ are pairwise strongly orthogonal martingales, and their predictable quadratic variation processes are $\left\langle H_{t}^{i}, H_{t}^{j}\right\rangle=\delta_{i j} t, \delta_{i j}$ is an indicator function here. And $\left[H^{i}, H^{j}\right]_{t}-\left\langle H_{t}^{i}, H_{t}^{j}\right\rangle$ is an $\mathscr{F}_{t}$-martingale; for more details of the Teugels martingale, see Nualart and Schoutens [20].

In the following of this section, we shall assume some notations: for a Hilbert space $\mathscr{H}$,

$$
\begin{aligned}
& l^{2}(\mathscr{H}):=\left\{\phi \mid \mathscr{H}-\text { valued, } \sum_{i=1}^{\infty}\left\|\phi^{i}\right\|^{2}<\infty\right\} . \\
& L^{2}(\Omega, \mathscr{H}):=\{\xi \mid \\
& \left.\mathscr{H} \text { valued, } \mathscr{F}_{T}-\text { measurable, } E|\xi|^{2}<\infty\right\} . \\
& l^{2}(0, T ; \mathscr{H}):=\left\{\phi_{t}^{i} \mid l^{2}(\mathscr{H})-\right. \\
& \text { valued, } \left.\mathscr{F}_{t}-\text { measurable, } \sum_{i=1}^{\infty} E \int_{0}^{T}\left\|\phi_{t}^{i}\right\|^{2} \mathrm{~d} t<\infty\right\} . \\
& M^{2}(0, T ; \mathscr{H}):=\{\phi(\cdot) \mid \\
& \left.\mathscr{H}-\text { valued, } \mathscr{F}_{t}-\text { measurable, } E \int_{0}^{T}\left|\phi_{t}\right|^{2} \mathrm{~d} t<\infty\right\} .
\end{aligned}
$$

For the following FBSDEL,

$$
\left\{\begin{array}{l}
\mathrm{d} x_{t}=b\left(t, x_{t}, y_{t}, z_{t}, r_{t}\right) \mathrm{d} t+\sigma\left(t, x_{t}, y_{t}, z_{t}, r_{t}\right) \mathrm{d} B_{t}+\sum_{i=1}^{\infty} g^{i}\left(t, x_{t-}, y_{t-}, z_{t}, r_{t}\right) \mathrm{d} H_{t}^{i}, \\
-\mathrm{d} y_{t}=f\left(t, x_{t}, y_{t}, z_{t}, r_{t}\right) \mathrm{d} t-z_{t} \mathrm{~d} B_{t}-\sum_{i=1}^{\infty} r_{t}^{i} \mathrm{~d} H_{t}^{i}, \\
x_{0}=a, y_{T}=\Phi\left(x_{T}\right),
\end{array}\right.
$$


where $\left(x_{t}, y_{t}, z_{t}, r_{t}\right)$ take the value in $\Omega \times[0, T] \times R^{n} \times R^{m} \times$ $R^{m \times d} \times l^{2}\left(R^{m}\right)$ and mappings $b, \sigma, g$, and $f$ take the value in $R^{n}, R^{n \times d}, l^{2}\left(R^{n}\right)$, and $R^{m}$, respectively. Convenient for writing, set column vector $\alpha=(x, y, z)^{T}$ and $A(t, \alpha, r)=\left(-M^{\tau} f(t, \alpha, r), M b(t, \alpha, r), M \sigma(t, \alpha, r)\right)^{T}$, where $M$ is a $m \times n$ full rank matrix.

\section{Assumption 1}

(i) All mappings in equation (1) are uniformly Lipschitz continuous in their own arguments, respectively.

(ii) For all $(\omega, t) \in \Omega \times[0, T], \quad l(\omega, t, 0,0,0,0)$ $\in M^{2}\left(0, T ; R^{n+m+m \times d}\right) \times l^{2}\left(0, T ; R^{m}\right)$ for $l=b, f, \sigma$, respectively, and $g(\omega, t, 0,0,0,0) \in l^{2}\left(R^{n}\right)$.

(iii) $\langle\Phi(\bar{x})-\Phi(x), M(\bar{x}-x)\rangle \geq \beta|M \hat{x}|^{2}$.

(iv) $\langle A(t, \bar{\alpha}, \bar{r})-A(t, \alpha, r), \bar{\alpha}-\alpha\rangle+\sum_{i=1}^{\infty}\left\langle M{\widehat{g^{i}}}^{i}, \widehat{r^{i}}\right\rangle \leq-\mu$ ${ }_{1}|M \hat{x}|^{2}-\mu_{2}\left(\left|M^{\tau} \hat{y}\right|^{2}+\left|M^{\tau} \widehat{z}\right|^{2}+\sum_{i=1}^{\infty}\left\|M^{\tau} \hat{r}^{i}\right\|^{2}\right)$, where $\bar{\alpha}=(\bar{x}, \bar{y}, \bar{z}), \hat{x}=\bar{x}-x, \hat{y}=\bar{y}-y, \widehat{z}=\bar{z}-z$, $\widehat{r^{i}}=\bar{r}^{i}-r^{i}, \widehat{g}^{i}=g^{i}(t, \bar{\alpha}, \bar{r})-g^{i}(t, \alpha, r) . \mu_{1}, \mu_{2}$, and $\beta$ are nonnegative constants which satisfied $\mu_{1}+\mu_{2}>0, \mu_{2}+\beta>0$, and $\mu_{1}>0, \beta>0\left(\right.$ resp. $\left.\mu_{2}>0\right)$ when $m>n$ (resp. $n>m)$.

Then, the following existence and uniqueness of the solution conclusion holds.

Lemma 1. There exists a unique solution in $M^{2}(0, T ; \mathscr{H})$ satisfying FBSDEL (1) under Assumption 1.

The detailed certification process of this conclusion can be seen in [24].

\section{Stochastic Maximum Principle}

In this section, for any given admissible control $u(\cdot)$, we consider the following stochastic control system:

$$
\left\{\begin{array}{l}
\mathrm{d} x_{t}=b\left(t, x_{t}, u_{t}\right) \mathrm{d} t+\sigma\left(t, x_{t}\right) \mathrm{d} B_{t}+\sum_{i=1}^{\infty} g^{i}\left(t, x_{t-}\right) \mathrm{d} H_{t}^{i}, \\
-\mathrm{d} y_{t}=f\left(t, x_{t}, y_{t}, z_{t}, r_{t}, u_{t}\right) \mathrm{d} t-z_{t} \mathrm{~d} B_{t}-\sum_{i=1}^{\infty} r_{t}^{i} \mathrm{~d} H_{t}^{i}, \\
x_{0}=a, y_{T}=\Phi\left(x_{T}\right), t \in[0, T],
\end{array}\right.
$$

where $a \in R^{n}$ is given. An admissible control $u(\cdot) \in M^{2}\left(0, T ; R^{p}\right)$ is an $\mathscr{F}_{t}$-predictable process which takes values in a nonempty subset $U$ of $R^{p} ; U_{\text {ad }}$ is the set of all admissible controls.

And the performance criterion is

$$
J(u)=E \gamma\left(y_{0}\right)
$$

where $\gamma: R^{m} \longrightarrow R$ is a given Frechet differential function.

Our optimal control problem amounts to determining an admissible control $u^{*} \in U_{\text {ad }}$ such that

$$
J\left(u^{*}(\cdot)\right)=\inf _{u(\cdot) \in U_{\mathrm{ad}}} J(u(\cdot)) .
$$

In order to get the necessary conditions for the optimal control, we assume $u_{t}^{*}$ is the optimal control, and the corresponding solution of (2) is recorded as $\left(x_{t}^{*}, y_{t}^{*}, z_{t}^{*}, r_{t}^{*}\right)$ and introduce the "spike variational control" as follows:

$$
u_{t}^{\varepsilon}= \begin{cases}v_{t}, & \tau \leq t \leq \tau+\varepsilon, \\ u_{t}^{*}, & \text { otherwise }\end{cases}
$$

and $\left(x_{t}^{\varepsilon}, y_{t}^{\varepsilon}, z_{t}^{\varepsilon}, r_{t}^{\varepsilon}\right)$ are the state trajectories of $u_{t}^{\varepsilon}$; here, $v_{t}$ is an arbitrary admissible control and $\varepsilon$ is a sufficiently small constant.

We also need the following assumption and variational equation (6).

\section{Assumption 2}

(i) $b, f, g, \sigma, \Phi$, and $\gamma$ are continuously differentiable with respect to $(x, y, z, r, u)$, and the derivatives are all bounded.

(ii) There exists a constant $C>0$, and it holds that $\left|\gamma_{y}\right| \leq C(1+|y|)$

$$
\left\{\begin{array}{l}
\mathrm{d} X_{t}=\left[b_{x}(t) X_{t}+b\left(t, u_{t}^{\varepsilon}\right)-b\left(t, u_{t}^{*}\right)\right] \mathrm{d} t+\sigma_{x}(t) X_{t} \mathrm{~d} B_{t}+\sum_{i=1}^{\infty} g_{x}^{i}(t) X_{t} \mathrm{~d} H_{t}^{i} \\
-\mathrm{d} Y_{t}=\left[f_{x}(t) X_{t}+f_{y}(t) Y_{t}+f_{z}(t) Z_{t}+f_{r}(t) R_{t}+f\left(t, u_{t}^{\varepsilon}\right)-f\left(t, u_{t}^{*}\right)\right] \mathrm{d} t \\
-Z_{t} \mathrm{~d} B_{t}-\sum_{i=1}^{\infty} R_{t}^{i} \mathrm{~d} H_{t}^{i} \\
X_{0}=0 \\
Y_{T}=\Phi_{x}(t) X_{T}
\end{array}\right.
$$


Here, $\quad b_{x}(t)=b_{x}\left(t, x_{t}^{*}, u_{t}^{*}\right), \quad \sigma_{x}(t)=\sigma_{x}\left(t, x_{t}^{*}\right), \quad g_{x}^{i}(t)=$ $g_{x}^{i}\left(t, x_{t}^{*}\right), \quad b\left(t, u_{t}^{\varepsilon}\right)=b\left(t, x_{t}^{\varepsilon}, u_{t}^{\varepsilon}\right), \quad b\left(t, u_{t}^{*}\right)=b \quad\left(t, x_{t}^{*}, u_{t}^{*}\right)$, $f_{w}(t)=f_{w}\left(t, x_{t}^{*}, y_{t}^{*}, z_{t}^{*}, r_{t}^{*}, u_{t}^{*}\right), \quad(w=x, y, z \cdot r), \quad f\left(t, u_{t}^{\varepsilon}\right)=$ $f\left(x_{t}^{\varepsilon}, y_{t}^{\varepsilon}, z_{t}^{\varepsilon}, r_{t}^{\varepsilon}, u_{t}^{\varepsilon}\right)$, and $f\left(t, u_{t}^{*}\right)=f\left(x_{t}^{*}, y_{t}^{*}, z_{t}^{*}, r_{t}^{*}, u_{t}^{*}\right)$.

Lemma 2. Suppose Assumptions 1 and 2 hold; for the firstorder variation $X, Y, Z, R$, we have the following estimations:

$$
\begin{aligned}
& \sup _{0 \leq t \leq T} E\left|X_{t}\right|^{2} \leq C \varepsilon^{2}, \\
& \sup _{0 \leq t \leq T} E\left|X_{t}\right|^{4} \leq C \varepsilon^{4}, \\
& \sup _{0 \leq t \leq T} E\left|Y_{t}\right|^{2} \leq C \varepsilon^{2}, \\
& \sup _{0 \leq t \leq T} E\left|Y_{t}\right|^{4} \leq C \varepsilon^{4},
\end{aligned}
$$

$$
\begin{array}{r}
\sup _{0 \leq t \leq T} E \int_{0}^{T}\left|Z_{t}\right|^{2} \mathrm{~d} s \leq C \varepsilon^{2}, \\
\sup _{0 \leq t \leq T} E\left(\int_{0}^{T}\left|Z_{t}\right|^{2} \mathrm{~d} s\right)^{2} \leq C \varepsilon^{4}, \\
\sup _{0 \leq t \leq T} E \int_{0}^{T}\left\|R_{t}\right\|^{2} \mathrm{~d} s \leq C \varepsilon^{2}, \\
\sup _{0 \leq t \leq T} E\left(\int_{0}^{T}\left\|R_{t}\right\|^{2} \mathrm{~d} s\right)^{2} \leq C \varepsilon^{4} .
\end{array}
$$

Proof. We first prove inequations (7) and (8). For the forward part of the first-order variation equation, we have

$$
\begin{aligned}
E\left|X_{t}\right|^{2} & =E\left\{\int_{0}^{t}\left[b_{x}(s) X_{s}+b\left(s, u_{s}^{\varepsilon}\right)-b\left(s, u_{s}^{*}\right)\right] \mathrm{d} s+\sigma_{x}(s) X_{s} \mathrm{~d} B_{s}+\sum_{i=1}^{\infty} g_{x}^{i}(s) X_{s} \mathrm{~d} H_{s}^{i}\right\}^{2} \\
& \leq 4\left\{E\left(\int_{0}^{t} b_{x}(s) X_{s} \mathrm{~d} s\right)^{2}+E\left(\int_{0}^{t}\left[b\left(s, u_{s}^{\varepsilon}\right)-b\left(s, u_{s}^{*}\right)\right] \mathrm{d} s\right)^{2}+E \int_{0}^{t}\left[\sigma_{x}(s) X_{s}\right]^{2} \mathrm{~d} s+E \int_{0}^{t}\left[\sum_{i=1}^{\infty} g_{x}^{i}(s) X_{s}\right]^{2} \mathrm{~d} s\right\} \\
& \leq 12 C^{2} T E \int_{0}^{t} X_{s}^{2} \mathrm{~d} s+4 E\left(\int_{0}^{t}\left[b\left(s, u_{s}^{\varepsilon}\right)-b\left(s, u_{s}^{*}\right)\right] \mathrm{d} s\right)^{2} .
\end{aligned}
$$

Applying Gronwall's inequation, we have

$$
E\left|X_{t}\right|^{2} \leq C \varepsilon^{2}, \quad \text { for all } t \in[0, T] .
$$

Similarly, (8) holds.

We next estimate $Y_{t}, Z_{t}$, and $R_{t}$; the backward part of the first-order variation equation can be rewritten as

$$
\begin{aligned}
Y_{t}+\int_{t}^{T} Z_{s} \mathrm{~d} B_{s}+\int_{t}^{T} \sum_{i=1}^{\infty} R_{s}^{i} \mathrm{~d} H_{s}^{i}= & \Phi_{x}(t) X_{T}+\int_{t}^{T}\left[f_{x}(t) X_{t}\right. \\
& +f_{y}(t) Y_{t}+f_{z}(t) Z_{t} \\
& +f_{r}(t) R_{t} \\
& \left.+f\left(t, u_{t}^{\varepsilon}\right)-f\left(t, u_{t}^{*}\right)\right] \mathrm{d} t .
\end{aligned}
$$

Squaring both sides of (17) and using the fact of

$$
\begin{array}{r}
E Y_{t} \int_{t}^{T} Z_{s} \mathrm{~d} B_{s}=0, \\
E Y_{t} \int_{t}^{T} \sum_{i=1}^{\infty} R_{s}^{i} \mathrm{~d} H_{s}^{i}=0, \\
E \int_{t}^{T} Z_{s} \mathrm{~d} B_{s} \int_{t}^{T} \sum_{i=1}^{\infty} R_{s}^{i} d H_{s}^{i}=0,
\end{array}
$$

we get

$$
\begin{aligned}
& E\left|Y_{t}\right|^{2}+E \int_{t}^{T} Z_{s}^{2} \mathrm{~d} s+E \int_{t}^{T}\left(\sum_{i=1}^{\infty} R_{s}^{i}\right)^{2} \mathrm{~d} H_{s}^{i} \\
& =E\left\{\Phi_{x}(t) X_{T}+\int_{t}^{T}\left[f_{x}(t) X_{t}+f_{y}(t) Y_{t}+f_{z}(t) Z_{t}+f_{r}(t) R_{t}+f\left(t, u_{t}^{\varepsilon}\right)-f\left(t, u_{t}^{*}\right)\right] \mathrm{d} t\right\}^{2} \\
& \leq 6 C^{2} E X_{T}^{2}+6 C^{2} T E \int_{t}^{T} X_{s}^{2} \mathrm{~d} s+6 C^{2} T E \int_{t}^{T} Y_{s}^{2} \mathrm{~d} s+6 C^{2}(T-t) E \int_{t}^{T} Z_{s}^{2} \mathrm{~d} s \\
& \quad+6 C^{2}(T-t) E \int_{t}^{T}\left(\sum_{i=1}^{\infty} R_{s}^{i}\right)^{2} \mathrm{~d} s+6 E\left(\int_{t}^{T}\left(f\left(s, u_{s}^{\varepsilon}\right)-f\left(s, u_{s}^{*}\right)\right) \mathrm{d} s\right)^{2}
\end{aligned}
$$


When $t \in[T-\delta, T]$ with $\delta=1 / 12 C^{2}$, we have

$$
\begin{gathered}
E\left|Y_{t}\right|^{2}+\frac{1}{2} E \int_{t}^{T} Z_{s}^{2} \mathrm{~d} s+\frac{1}{2} E \int_{t}^{T}\left(\sum_{i=1}^{\infty} R_{s}^{i}\right)^{2} \mathrm{~d} H_{s}^{i} \\
\leq 6 C^{2} E X_{T}^{2}+6 C^{2} T E \int_{t}^{T} X_{s}^{2} \mathrm{~d} s+6 C^{2} T E \\
\cdot \int_{t}^{T} Y_{s}^{2} \mathrm{~d} s+6 E\left(\int_{t}^{T}\left(f\left(s, u_{s}^{\varepsilon}\right)-f\left(s, u_{s}^{*}\right)\right) \mathrm{d} s\right)^{2} .
\end{gathered}
$$

Applying Gronwall's inequation, we have

$$
\begin{aligned}
& \sup _{0 \leq t \leq T} E\left|Y_{t}\right|^{2} \leq C \varepsilon^{2}, \quad t \in[T-\delta, T] ; \\
& \sup _{0 \leq t \leq T} E \int_{0}^{T}\left|Z_{t}\right|^{2} \mathrm{~d} s \leq C \varepsilon^{2}, \quad t \in[T-\delta, T] ; \\
& \sup _{0 \leq t \leq T} E \int_{0}^{T}\left\|R_{t}\right\|^{2} \mathrm{~d} s \leq C \varepsilon^{2}, \quad t \in[T-\delta, T] .
\end{aligned}
$$

Consider the BSDE of the first-order variation equation in the interval $[t, T-\delta]$ :

$$
\begin{aligned}
Y_{t} & +\int_{t}^{T-\delta} Z_{s} \mathrm{~d} B_{s}+\int_{t}^{T-\delta} \sum_{i=1}^{\infty} R_{s}^{i} \mathrm{~d} H_{s}^{i}=Y_{T-\delta} \\
& +\int_{t}^{T-\delta}\left[f_{x}(t) X_{t}+f_{y}(t) Y_{t}+f_{z}(t) Z_{t}\right. \\
& \left.+f_{r}(t) R_{t}+f\left(t, u_{t}^{\varepsilon}\right)-f\left(t, u_{t}^{*}\right)\right] \mathrm{d} t .
\end{aligned}
$$

Thus,

$$
\begin{aligned}
& E\left|Y_{t}\right|^{2}+E \int_{t}^{T-\delta} Z_{s}^{2} \mathrm{~d} s+E \int_{t}^{T-\delta}\left(\sum_{i=1}^{\infty} R_{s}^{i}\right)^{2} \mathrm{~d} H_{s}^{i}, \\
& =E\left\{Y_{T-\delta}+\int_{t}^{T-\delta}\left[f_{x}(t) X_{t}+f_{y}(t) Y_{t}+f_{z}(t) Z_{t}+f_{r}(t) R_{t}\right.\right. \\
& \left.\left.\quad+f\left(t, u_{t}^{\varepsilon}\right)-f\left(t, u_{t}^{*}\right)\right] \mathrm{d} t\right\}^{2} \\
& \leq 6 C^{2} E X_{T}^{2}+6 C^{2} T E \int_{t}^{T-\delta} X_{s}^{2} \mathrm{~d} s+6 C^{2} T E \int_{t}^{T-\delta} Y_{s}^{2} \mathrm{~d} s \\
& \quad+6 C^{2}(T-t) E \int_{t}^{T-\delta} Z_{s}^{2} \mathrm{~d} s+6 C^{2}(T-t) E \\
& \quad \cdot \int_{t}^{T-\delta}\left(\sum_{i=1}^{\infty} R_{s}^{i}\right)^{2} \mathrm{~d} s+6 E\left(\int_{t}^{T-\delta}\left(f\left(s, u_{s}^{\varepsilon}\right)-f\left(s, u_{s}^{*}\right)\right) \mathrm{d} s\right)^{2} .
\end{aligned}
$$

So, when $t \in[T-2 \delta, T]$ with $\delta=1 / 12 C^{2}$, we have

$$
\begin{aligned}
& \sup _{0 \leq t \leq T} E\left|Y_{t}\right|^{2} \leq C \varepsilon^{2}, \quad t \in[T-2 \delta, T], \\
& \sup _{0 \leq t \leq T} E \int_{0}^{T}\left|Z_{t}\right|^{2} \mathrm{~d} s \leq C \varepsilon^{2}, \quad t \in[T-2 \delta, T], \\
& \sup _{0 \leq t \leq T} E \int_{0}^{T}\left\|R_{t}\right\|^{2} \mathrm{~d} s \leq C \varepsilon^{2}, \quad t \in[T-2 \delta, T] .
\end{aligned}
$$

After a finite number of iterations, (9), (11), and (13) are obtained. And (10), (12), and (14) can be proved by using a similar method and the following inequalities: $E\left(\int_{t}^{T} Z_{s} \mathrm{~d} B_{s}\right)^{4} \geq \beta_{1} E\left(\int_{t}^{T} Z_{s}^{2} \mathrm{~d} s\right)^{2}, \beta_{1}>0 ;$

$E\left(\int_{t}^{T} \sum_{i=1}^{\infty} R_{s}^{i} \mathrm{~d} H_{s}^{i}\right)^{4} \geq \beta_{2} E\left(\int_{t}^{T}\left(\sum_{i=1}^{\infty} R_{s}^{i}\right)^{2} \mathrm{~d} s\right)^{2}, \beta_{2}>0$.

Lemma 3. Under hypothesis, Assumptions 1 and 2, it holds the following four estimations:

$$
\sup _{0 \leq t \leq T} E\left|x_{t}^{\varepsilon}-x_{t}^{*}-X_{t}\right|^{2} \leq C_{\varepsilon} \varepsilon^{2}, \quad C_{\varepsilon} \longrightarrow 0 \text {, when } \varepsilon \longrightarrow 0,
$$

$$
\sup _{0 \leq t \leq T} E\left|y_{t}^{\varepsilon}-y_{t}^{*}-Y_{t}\right|^{2} \leq C_{\varepsilon} \varepsilon^{2}, \quad C_{\varepsilon} \longrightarrow 0 \text {, when } \varepsilon \longrightarrow 0 \text {, }
$$

$E \int_{0}^{T}\left|z_{t}^{\varepsilon}-z_{t}^{*}-Z_{t}\right|^{2} \mathrm{~d} s \leq C_{\varepsilon} \varepsilon^{2}, \quad C_{\varepsilon} \longrightarrow 0$, when $\varepsilon \longrightarrow 0$,

$E \int_{t}^{T}\left\|r_{t}^{\varepsilon}-r_{t}^{*}-R_{t}\right\|^{2} \mathrm{~d} s \leq C_{\varepsilon} \varepsilon^{2}, \quad C_{\varepsilon} \longrightarrow 0$, when $\varepsilon \longrightarrow 0$.

Proof. To prove (25), we observe that

$$
\begin{aligned}
& \int_{0}^{t} b\left(s, x_{s}^{*}+X_{s}, u_{s}^{\varepsilon}\right) \mathrm{d} s+\int_{0}^{t} \sigma\left(s, x_{s}^{*}+X_{s}\right) \mathrm{d} B_{s} \\
& +\int_{0}^{t} \sum_{i=1}^{\infty} g^{i}\left(s, x_{s-}^{*}+X_{s-}\right) \mathrm{d} H_{s}^{i} \\
& =\int_{0}^{t}\left[b\left(s, x_{s}^{*}, u_{s}^{\varepsilon}\right)+\int_{0}^{1} b_{x}\left(s, x_{s}^{*}+\lambda X_{s}, u_{s}^{\varepsilon}\right) \mathrm{d} \lambda X_{s}\right] \mathrm{d} s \\
& +\int_{0}^{t}\left[\sigma\left(s, x_{s}^{*}\right)+\int_{0}^{1} \sigma_{x}\left(s, x_{s}^{*}+\lambda X_{s}\right) \mathrm{d} \lambda X_{s}\right] \mathrm{d} B_{s} \\
& +\int_{0}^{t}\left[\sum_{i=1}^{\infty} g^{i}\left(s, x_{s}^{*}\right)+\int_{0}^{1} \sum_{i=1}^{\infty} g_{x}^{i}\left(s, x_{s}^{*}+\lambda X_{s}\right) d \lambda X_{s}\right] \mathrm{d} H_{s}^{i} \\
& =\int_{0}^{t} b\left(s, x_{s}^{*}, u_{s}^{*}\right) \mathrm{d} s+\int_{0}^{t} \sigma\left(s, x_{s}^{*}\right) \mathrm{d} B_{s}+\int_{0}^{t} \sum_{i=1}^{\infty} g^{i}\left(s, x_{s}^{*}\right) \mathrm{d} H_{s}^{i} \\
& +\int_{0}^{t} b_{x}\left(s, x_{s}^{*}, u_{s}^{*}\right) X_{s} \mathrm{~d} s+\int_{0}^{t} \sigma_{x}\left(s, x_{s}^{*}\right) X_{s} \mathrm{~d} B_{s} \\
& +\int_{0}^{t} \sum_{i=1}^{\infty} g_{x}^{i}\left(s, x_{s-}^{*}\right) \mathrm{d} H_{s}^{i} \\
& +\int_{0}^{t}\left[b\left(s, x_{s}^{*}, u_{s}^{\varepsilon}\right)-b\left(s, x_{s}^{*}, u_{s}^{*}\right)\right] \mathrm{d} s+\int_{0}^{t} A^{\varepsilon} \mathrm{d} s \\
& +\int_{0}^{t} B^{\varepsilon} \mathrm{d} B_{s}+\int_{0}^{t} C^{\varepsilon} \mathrm{d} H_{s}^{i} \\
& =x_{t}^{*}-x_{0}+X_{t}+\int_{0}^{t} A^{\varepsilon} \mathrm{d} s+\int_{0}^{t} B^{\varepsilon} \mathrm{d} B_{s}+\int_{0}^{t} C^{\varepsilon} \mathrm{d} H_{s}^{i},
\end{aligned}
$$


where

$$
\begin{aligned}
& A^{\varepsilon}=\int_{0}^{1}\left[b_{x}\left(s, x_{s}^{*}+\lambda X_{s}, u_{s}^{\varepsilon}\right)-b_{x}\left(s, x_{s}^{*}, u_{s}^{*}\right)\right] \mathrm{d} \lambda X_{s}, \\
& B^{\varepsilon}=\int_{0}^{1}\left[\sigma_{x}\left(s, x_{s}^{*}+\lambda X_{s}\right)-\sigma_{x}\left(s, x_{s}^{*}\right)\right] \mathrm{d} \lambda X_{s}, \\
& C^{\varepsilon}=\int_{0}^{1}\left[\sum_{i=1}^{\infty}\left(g_{x}^{i}\left(s, x_{s}^{*}+\lambda X_{s}\right)-g_{x}^{i}\left(s, x_{s}^{*}\right)\right)\right] \mathrm{d} \lambda X_{s} .
\end{aligned}
$$

It follows easily from Lemma 2 that

$$
\sup _{0 \leq t \leq T} E\left[\left(\int_{0}^{t} A^{\varepsilon} \mathrm{d} s\right)^{2}+\left(\int_{0}^{t} B^{\varepsilon} \mathrm{d} B_{s}\right)^{2}+\left(\int_{0}^{t} C^{\varepsilon} \mathrm{d} H_{s}^{i}\right)^{2}\right]=o\left(\varepsilon^{2}\right) .
$$

Since

$$
x_{t}^{\varepsilon}-x_{0}=\int_{0}^{t} b\left(s, x_{s}^{\varepsilon}, u_{s}^{\varepsilon}\right) \mathrm{d} s+\int_{0}^{t} \sigma\left(s, x_{s}^{\varepsilon}\right) \mathrm{d} B_{s}+\int_{0}^{t} \sum_{i=1}^{\infty} g^{i}\left(s, x_{s-}^{\varepsilon}\right) \mathrm{d} H_{s^{i}}^{i}
$$

then

$$
\begin{aligned}
x_{t}^{\varepsilon}-x_{t}^{*}-X_{t}= & \int_{0}^{t}\left[b\left(s, x_{s}^{\varepsilon}, u_{s}^{\varepsilon}\right)-b\left(s, x_{s}^{*}+X_{s}, u_{s}^{\varepsilon}\right)\right] \mathrm{d} s \\
& +\int_{0}^{t}\left[\sigma\left(s, x_{s}^{\varepsilon}\right)-\sigma\left(s, x_{s}^{*}+X_{s}\right)\right] \mathrm{d} B_{s} \\
& +\int_{0}^{t} \sum_{i=1}^{\infty}\left(g^{i}\left(s, x_{s-}^{\varepsilon}\right)-g^{i}\left(s, x_{s-}^{*}+X_{s-}\right)\right) \mathrm{d} H_{s}^{i} \\
& +\int_{0}^{t} A^{\varepsilon} \mathrm{d} s+\int_{0}^{t} B^{\varepsilon} \mathrm{d} B_{s}+\int_{0}^{t} C^{\varepsilon} \mathrm{d} H_{s}^{i} \\
= & \int_{0}^{t} D^{\varepsilon}\left(x_{s}^{\varepsilon}-x_{s}^{*}-X_{s}\right) \mathrm{d} s+\int_{0}^{t} E^{\varepsilon}\left(x_{s}^{\varepsilon}-x_{s}^{*}-X_{s}\right) \mathrm{d} B_{s} \\
& +\int_{0}^{t} F^{\varepsilon}\left(x_{s}^{\varepsilon}-x_{s}^{*}-X_{s}\right) \mathrm{d} H_{s}^{i},
\end{aligned}
$$

with $\quad D^{\varepsilon}=\int_{0}^{1} b_{x}\left(s, x_{s}^{*}+X_{s}+\lambda\left(x_{s}^{\varepsilon}-x_{s}^{*}-X_{s}\right), u_{s}^{\varepsilon}\right) \mathrm{d} \lambda$, $E^{\varepsilon}=\int_{0}^{1} \sigma_{x}\left(s, x_{s}^{*}+X_{s}+\lambda\left(x_{s}^{\varepsilon}-x_{s}^{*}-X_{s}\right)\right) \mathrm{d} \lambda$, and $F^{\varepsilon}=\int_{0}^{1}$ $\sum_{i=1}^{\infty} g_{x}^{i}\left(s, x_{s}^{*}+X_{s}+\lambda\left(x_{s}^{\varepsilon}-x_{s}^{*}-X_{s}\right)\right) \mathrm{d} \lambda$.

By Gronwall's inequation, we have

$$
\sup _{0 \leq t \leq T} E\left|x_{t}^{\varepsilon}-x_{t}^{*}-X_{t}\right|^{2} \leq C_{\varepsilon} \varepsilon^{2}, \quad C_{\varepsilon} \longrightarrow 0 \text {, when } \varepsilon \longrightarrow 0 \text {. }
$$

Next, we prove (26), (27),, (28); it can be easily checked that

$$
\begin{aligned}
& -\int_{t}^{T} f\left(s, x_{s}^{*}+X_{s}, y_{s}^{*}+Y_{s}, z_{s}^{*}+Z_{s}, r_{s}^{*}+R_{s}, u_{s}^{\varepsilon}\right) \mathrm{d} s \\
& +\int_{t}^{T}\left(s, z_{s}^{*}+Z_{s}\right) \mathrm{d} B_{s}+\int_{t}^{T} \sum_{i=1}^{\infty} g^{i}\left(s, x_{s-}^{*}+X_{s-}\right) \mathrm{d} H_{s}^{i} \\
& =\Phi\left(x_{T}^{*}\right)-y_{t}^{*}+\Phi_{x}\left(x_{T}^{*}\right) X_{T}-Y_{t}-\int_{t}^{T} G^{\varepsilon} \mathrm{d} s .
\end{aligned}
$$

Here,

$$
\begin{aligned}
G^{\varepsilon}= & \int_{0}^{1}\left(f _ { x } \left(s, x_{s}^{*}+\lambda X_{s}, y_{s}^{*}+\lambda Y_{s}, z_{s}^{*}\right.\right. \\
& \left.\left.+\lambda Z_{s}, r_{s}^{*}+\lambda R_{s}, u_{s}^{\varepsilon}\right)-f_{x}(s)\right) \mathrm{d} \lambda X_{s} \\
& +\int_{0}^{1}\left(f _ { y } \left(s, x_{s}^{*}+\lambda X_{s}, y_{s}^{*}+\lambda Y_{s}, z_{s}^{*}\right.\right. \\
& \left.\left.+\lambda Z_{s}, r_{s}^{*}+\lambda R_{s}, u_{s}^{\varepsilon}\right)-f_{y}(s)\right) \mathrm{d} \lambda Y_{s} \\
& +\int_{0}^{1}\left(f _ { z } \left(s, x_{s}^{*}+\lambda X_{s}, y_{s}^{*}+\lambda Y_{s}, z_{s}^{*}\right.\right. \\
& \left.\left.+\lambda Z_{s}, r_{s}^{*}+\lambda R_{s}, u_{s}^{\varepsilon}\right)-f_{z}(s)\right) \mathrm{d} \lambda Z_{s} \\
& +\int_{0}^{1}\left(f _ { r } \left(s, x_{s}^{*}+\lambda X_{s}, y_{s}^{*}+\lambda Y_{s}, z_{s}^{*}\right.\right. \\
& \left.\left.+\lambda Z_{s}, r_{s}^{*}+\lambda R_{s}, u_{s}^{\varepsilon}\right)-f_{r}(s)\right) \mathrm{d} \lambda R_{s} .
\end{aligned}
$$

Since

$$
\begin{aligned}
y_{t}^{\varepsilon}= & \Phi\left(x_{T}^{\varepsilon}\right)+\int_{t}^{T} f\left(s, x_{s}^{\varepsilon}, y_{s}^{\varepsilon}, z_{s}^{\varepsilon}, r_{s}^{\varepsilon}, u_{s}^{\varepsilon}\right) \mathrm{d} s-\int_{t}^{T} z_{s} \mathrm{~d} B_{s} \\
& -\int_{t}^{T} \sum_{i=1}^{\infty} r_{s}^{i, \varepsilon} \mathrm{d} H_{s}^{i},
\end{aligned}
$$

$$
\begin{aligned}
y_{t}^{\varepsilon}-y_{t}^{*}-Y_{t}= & \Phi\left(x_{T}^{\varepsilon}\right)-\Phi\left(x_{T}^{*}\right)-\Phi_{x}\left(x_{T}^{*}\right) X_{T}+\int_{t}^{T}\left[f\left(s, x_{s}^{\varepsilon}, y_{s}^{\varepsilon}, z_{s}^{\varepsilon}, r_{s}^{\varepsilon}, u_{s}^{\varepsilon}\right)-f\left(s, x_{s}^{*}+X_{s}, y_{s}^{*}+Y_{s}, z_{s}^{*}+Z_{s}, r_{s}^{*}+R_{s}, u_{s}^{\varepsilon}\right] \mathrm{d} s\right. \\
& -\int_{t}^{T}\left(z_{s}^{\varepsilon}-z_{s}^{*}-Z_{s}\right) \mathrm{d} B_{s}-\int_{t}^{T} \sum_{i=1}^{\infty}\left(r_{s}^{i, \varepsilon}-r_{s}^{i, *}-R_{s}\right) \mathrm{d} H_{s}^{i}+\int_{t}^{T} G^{\varepsilon} \mathrm{d} s
\end{aligned}
$$


Squaring both sides of the equation above, we get

$$
\begin{aligned}
& E\left|y_{t}^{\varepsilon}-y_{t}^{*}-Y_{t}\right|^{2}+E \int_{t}^{T}\left(z_{s}^{\varepsilon}-z_{s}^{*}-Z_{s}\right)^{2} \mathrm{~d} s-E \int_{t}^{T} \sum_{i=1}^{\infty}\left(r_{s}^{i, \varepsilon}-r_{s}^{i, *}-R_{s}\right)^{2} \mathrm{~d} s \\
& =E\left\{\left[f\left(s, x_{s}^{\varepsilon}, y_{s}^{\varepsilon}, z_{s}^{\varepsilon}, r_{s}^{\varepsilon}, u_{s}^{\varepsilon}\right)-f\left(s, x_{s}^{*}+X_{s}, y_{s}^{*}+Y_{s}, z_{s}^{*}+Z_{s}, r_{s}^{*}+R_{s}, u_{s}^{\varepsilon}\right)\right] \mathrm{d} s+\Phi\left(x_{T}^{\varepsilon}\right)-\Phi\left(x_{T}^{*}\right)-\Phi_{x}\left(x_{T}^{*}\right) X_{T}+\int_{t}^{T} G^{\varepsilon} \mathrm{d} s\right\}^{2} .
\end{aligned}
$$

From Lemma 2 and equation (25), we have

$$
\begin{array}{r}
\sup _{0 \leq t \leq T} E\left(\int_{t}^{T} G^{\varepsilon} \mathrm{d} s\right)^{2}=o\left(\varepsilon^{2}\right), \\
E\left[\Phi\left(x_{T}^{\varepsilon}\right)-\Phi\left(x_{T}^{*}\right)-\Phi_{x}\left(x_{T}^{*}\right) X_{T}\right]^{2}=o\left(\varepsilon^{2}\right) .
\end{array}
$$

Then, we can get (26), (27), and (28) by applying the iterative method to the above relations.

Lemma 4 (variational inequality). Under the conditions that Assumptions 1 and 2 are established, we can get the following variational inequality:

$$
E \gamma_{y}\left(y_{0}^{*}\right) Y_{0} \geq o(\varepsilon) .
$$

Proof. From the four estimations in Lemma 3, we have the following estimation:

$$
E\left[\gamma\left(y_{0}^{\varepsilon}\right)-\gamma\left(y_{0}^{*}+Y_{0}\right)\right]=o(\varepsilon) .
$$

Therefore,

$$
0 \leq E\left[\gamma\left(y_{0}^{*}+Y_{0}\right)-\gamma\left(y_{0}^{*}\right)\right]+o(\varepsilon)=E \gamma_{y}\left(y_{0}^{*}\right) Y_{0}+o(\varepsilon) .
$$

We introduce the following Hamiltonian function $H:[0, T] \times R^{n} \times R^{m} \times R^{m \times d} \times l^{2}\left(R^{m}\right) \times U \times R^{n} \times R^{m} \times$ $R^{n \times d} \times l^{2}\left(R^{n}\right)$ as

$$
\begin{aligned}
& H(t, x, y, z, r, u, p, q, w, k)=\langle p, b(t, x, u)\rangle+\langle w, \sigma(t, x)\rangle \\
& \quad+\langle k, g(t, x)\rangle-\langle q, f(t, x, y, z, r, u)\rangle,
\end{aligned}
$$

and the following adjoint equation

$$
\left\{\begin{array}{l}
\mathrm{d} q_{t}=H_{y}\left(u_{t}^{*}\right) \mathrm{d} t+H_{z}\left(u_{t}^{*}\right) \mathrm{d} B_{t}+\sum_{i=1}^{\infty} H_{r}^{i}\left(u_{t}^{*}\right) \mathrm{d} H_{t}^{i}, \\
-\mathrm{d} p_{t}=H_{x}\left(u_{t}^{*}\right) \mathrm{d} t-w_{t} \mathrm{~d} B_{t}-\sum_{i=1}^{\infty} k_{t-}^{i} \mathrm{~d} H_{t}^{i}, \\
q_{0}=\gamma_{y}\left(y_{0}^{*}\right), p_{T}=-\Phi_{x}\left(x_{T}^{*}\right) q_{T}, \quad t \in[0, T],
\end{array}\right.
$$

where

$$
\begin{aligned}
& H_{x}\left(u_{t}^{*}\right)=H_{x}\left(t, x_{t}^{*}, y_{t}^{*}, z_{t}^{*}, r_{t}^{*}, u_{t}^{*}, p_{t}, q_{t}, w_{t}, k_{t}\right), \\
& H_{y}\left(u_{t}^{*}\right)=H_{y}\left(t, x_{t}^{*}, y_{t}^{*}, z_{t}^{*}, r_{t}^{*}, u_{t}^{*}, p_{t}, q_{t}, w_{t}, k_{t}\right), \\
& H_{z}\left(u_{t}^{*}\right)=H_{z}\left(t, x_{t}^{*}, y_{t}^{*}, z_{t}^{*}, r_{t}^{*}, u_{t}^{*}, p_{t}, q_{t}, w_{t}, k_{t}\right), \\
& H_{r}\left(u_{t}^{*}\right)=H_{r}\left(t, x_{t}^{*}, y_{t}^{*}, z_{t}^{*}, r_{t}^{*}, u_{t}^{*}, p_{t}, q_{t}, w_{t}, k_{t}\right) .
\end{aligned}
$$

It is easily check that adjoint equation (45) has a unique solution quartet $\left(p_{t}, q_{t}, w_{t}, k_{t}\right) \in M^{2}\left(0, T ; R^{n+m+m \times d}\right) \times$ $l^{2}\left(0, T ; R^{m}\right)$.

Then, we get the main result of this section.

Theorem 1. Let hypothesis, Assumptions 1 and2, hold; $u_{t}^{*}$ is an optimal control, and the corresponding optimal state trajectories are $\left(x_{t}^{*}, y_{t}^{*}, z_{t}^{*}, r_{t}^{*}\right)$; let $\left(p_{t}, q_{t}, w_{t}, k_{t}\right)$ be the solution of adjoint equation (45); and then, for each admissible control $u_{t} \in U_{a d}[0, T]$, we have

$$
\begin{aligned}
& H\left(t, x_{t}^{*}, y_{t}^{*}, z_{t}^{*}, r_{t}^{*}, u_{t}, p_{t}, q_{t}, w_{t}, k_{t}\right) \\
& \quad \geq H\left(t, x_{t}^{*}, y_{t}^{*}, z_{t}^{*}, r_{t}^{*}, u_{t}^{*}, p_{t}, q_{t}, w_{t}, k_{t}\right) \text { a.s.a.e.. }
\end{aligned}
$$

Proof. Applying It $\widehat{o}$ 's formula to $\langle p, X\rangle$ and $\langle q, Y\rangle$, it follows from (6), (45), and the variational inequality that

$$
\begin{aligned}
& E \int_{0}^{T}\left[H\left(t, x_{t}, y_{t}, z_{t}, r_{t}, u_{t}^{\varepsilon}, p_{t}, q_{t}, w_{t}, k_{t}\right)\right. \\
& \left.\quad-H\left(t, x_{t}, y_{t}, z_{t}, r_{t}, u_{t}^{*}, p_{t}, q_{t}, w_{t}, k_{t}\right)\right] \mathrm{d} t=E \gamma_{y}\left(y_{0}^{*}\right) Y_{0} \geq o(\varepsilon) .
\end{aligned}
$$

By the definition of $\boldsymbol{u}_{t}^{\varepsilon}$, we know that, for any $u \in U_{\text {ad }}[0, T]$, the following inequation holds:

$$
\begin{aligned}
& E\left[H\left(t, x_{t}, y_{t}, z_{t}, r_{t}, u_{t}^{\varepsilon}, p_{t}, q_{t}, w_{t}, k_{t}\right)\right. \\
& \left.\quad-H\left(t, x_{t}, y_{t}, z_{t}, r_{t}, u_{t}^{*}, p_{t}, q_{t}, w_{t}, k_{t}\right)\right] \geq 0 .
\end{aligned}
$$

Then, (47) can be easily checked.

\section{Stochastic Control Problem with State Constraints}

In this part, we are going to discuss stochastic control problems with state constraints in control system (2). Specifically, the initial state constraints and final state constraints are as follows:

$$
\begin{aligned}
& E G_{1}\left(x_{T}\right)=0, \\
& E G_{0}\left(y_{0}\right)=0,
\end{aligned}
$$


where $G_{1}: R^{n} \longrightarrow R^{n_{1}}\left(n_{1}<n\right), G_{0}: R^{m} \longrightarrow R^{m_{1}}\left(m_{1}<m\right)$. Our optimal control problem is to find $u^{*} \in U_{\text {ad }}$ such that

$$
J\left(u^{*}(\cdot)\right)=\inf _{u(\cdot) \in U_{\mathrm{ad}}} J(u(\cdot)) \text {, }
$$

subject to the state constraints (50). In the following, we will apply Ekeland's variational principle to solve this optimal control problem. Firstly, we need the following assumptions.

Assumption 3. Control domain $U$ is assumed to be closed, the mappings in state constraints $G_{1}, G_{0}$ are continuously differentiable, and $G_{1 x}, G_{0 y}$ are bounded.

For $\forall u_{1}(\cdot), u_{2}(\cdot) \in U_{\text {ad }}$, let

$$
d\left(u_{1}(\cdot), u_{2}(\cdot)\right)=\left|\left\{E\left|u_{1}(\cdot)-u_{2}(\cdot)\right|^{2}>0 ; t \in[0, T]\right\}\right| \cdot
$$

Same as Section 3, we also assume $u_{t}^{*}$ be the optimal control, and the corresponding optimal state trajectories are $\left(x_{t}^{*}, y_{t}^{*}, z_{t}^{*}, r_{t}^{*}\right)$. In order to solve the constraint problem, we need the following penalty cost functional, for any $\rho>0$ :

$$
J_{\rho}(u)=\left\{\left|E\left[G_{1}\left(x_{T}\right)\right]\right|^{2}+\left|E\left[G_{0}\left(y_{0}\right)\right]\right|^{2}+\left|J(u)-J\left(u^{*}\right)+\rho\right|^{2}\right\}^{1 / 2} .
$$

It can be checked that $J_{\rho}(u): U_{\text {ad }} \longrightarrow R^{1}$ is continuous, and for any $u(\cdot) \in U_{a d}$,

$$
\begin{aligned}
J_{\rho}(u) & \geq 0, J_{\rho}\left(u^{*}\right)=\rho, \\
J_{\rho}\left(u^{*}\right) & \leq \inf _{u(\cdot) \in U_{\mathrm{ad}}} J_{\rho}(u)+\rho .
\end{aligned}
$$

It can be obtained by Ekeland's variational principle that there exists $u_{t}^{\rho} \in U_{\text {ad }}$ such that

$$
\begin{aligned}
& \text { (i) } J_{\rho}\left(u^{\rho}\right) \leq J_{\rho}\left(u^{*}\right)=\rho, \\
& \text { (ii) } d\left(u^{\rho}, u^{*}\right) \leq \sqrt{\rho}, \\
& \text { (iii) } J_{\rho}(v) \geq J_{\rho}\left(u^{\rho}\right)-\sqrt{\rho} d\left(u^{\rho}, v\right), \quad \text { for } \forall v(\cdot) \in U_{\mathrm{ad}} .
\end{aligned}
$$

For fixed $\rho$ and admissible control $u_{t}^{\rho}$, we define the spike variation as follows:

$$
u_{t}^{\rho, \varepsilon}= \begin{cases}v_{t}, & \tau \leq t \leq \tau+\varepsilon \\ u_{t}^{\rho}, & \text { otherwise, }\end{cases}
$$

for any $\varepsilon>0$, and it is easy to check from (iii) of (55) that

$$
J_{\rho}\left(u^{\rho, \varepsilon}\right)-J_{\rho}\left(u^{\rho}\right)+\sqrt{\rho} d\left(u^{\rho, \varepsilon}, u^{\rho}\right) \geq 0 .
$$

Let $\left(x_{t}^{\rho}, y_{t}^{\rho}, z_{t}^{\rho}, r_{t}^{\rho}\right)$ be the trajectories corresponding to $u_{t}^{\rho}$ and $\left(x_{t}^{\rho, \varepsilon}, y_{t}^{\rho, \varepsilon}, z_{t}^{\rho, \varepsilon}, r_{t}^{\rho, \varepsilon}\right)$ be the trajectories corresponding to $u_{t}^{\rho, \varepsilon}$. The variational equation we used in this section is the same as the one in Section 3, with $u_{t}^{*}=u_{t}^{\rho}$ and $\left(x_{t}^{*}, y_{t}^{*}, z_{t}^{*}, r_{t}^{*}\right)=\left(x_{t}^{\rho}, y_{t}^{\rho}, z_{t}^{\rho}, r_{t}^{\rho}\right)$. And we also assume that the solution of this variational equation is $\left(X_{t}^{\rho}, Y_{t}^{\rho}, Z_{t}^{\rho}, R_{t}^{\rho}\right)$. Similar to the approach in Lemmas 2 and 3, it can be shown that

$$
\begin{array}{cl}
\sup _{0 \leq t \leq T} E\left|x_{t}^{\rho, \varepsilon}-x_{t}^{\rho}-X_{t}^{\rho}\right|^{2} \leq C_{\varepsilon} \varepsilon^{2}, & C_{\varepsilon} \longrightarrow 0 \text {, when } \varepsilon \longrightarrow 0, \\
\sup _{0 \leq t \leq T} E\left|y_{t}^{\rho, \varepsilon}-y_{t}^{\rho}-Y_{t}^{\rho}\right|^{2} \leq C_{\varepsilon} \varepsilon^{2}, & C_{\varepsilon} \longrightarrow 0, \text { when } \varepsilon \longrightarrow 0, \\
E \int_{0}^{T}\left|z_{t}^{\rho, \varepsilon}-z_{t}^{\rho}-Z_{t}^{\rho}\right|^{2} \mathrm{~d} s \leq C_{\varepsilon} \varepsilon^{2}, & C_{\varepsilon} \longrightarrow 0 \text {, when } \varepsilon \longrightarrow 0, \\
E \int_{0}^{T}\left\|r_{t}^{\rho, \varepsilon}-r_{t}^{\rho}-R_{t}^{\rho}\right\|^{2} \mathrm{~d} s \leq C_{\varepsilon} \varepsilon^{2}, \quad C_{\varepsilon} \longrightarrow 0, \text { when } \varepsilon \longrightarrow 0 .
\end{array}
$$

Then, by (57), the following variational inequality holds:

$$
\begin{aligned}
& J_{\rho}\left(u^{\rho, \varepsilon}\right)-J_{\rho}\left(u^{\rho}\right)+\sqrt{\rho} d\left(u^{\rho, \varepsilon}, u^{\rho}\right) \\
& =\frac{J_{\rho}^{2}\left(u^{\rho, \varepsilon}\right)-J_{\rho}^{2}\left(u^{\rho}\right)}{J_{\rho}\left(u^{\rho, \varepsilon}\right)+J_{\rho}\left(u^{\rho}\right)}+\varepsilon \sqrt{\rho} \\
& =\left\langle h_{1}^{\rho, \varepsilon}, E\left[G_{1 x}\left(x_{T}^{\rho}\right)\right] X_{T}^{\rho}\right\rangle+\left\langle h_{0}^{\rho, \mathcal{\varepsilon}}, E\left[G_{0 y}\left(y_{0}^{\rho}\right)\right] Y_{0}^{\rho}\right\rangle \\
& \quad+h^{\rho, \varepsilon} E\left[\gamma_{y}\left(y_{0}^{\rho}\right) Y_{0}^{\rho}\right]+\varepsilon \sqrt{\rho}+o(\varepsilon),
\end{aligned}
$$

where

$$
\begin{aligned}
h_{1}^{\rho, \mathcal{\varepsilon}} & =\frac{2 E\left[G_{1}\left(x_{T}^{\rho}\right)\right]}{J_{\rho}\left(u^{\rho, \varepsilon}\right)+J_{\rho}\left(u^{\rho}\right)}, \\
h_{0}^{\rho, \mathcal{\varepsilon}} & =\frac{2 E\left[G_{0}\left(y_{0}^{\rho}\right)\right]}{J_{\rho}\left(u^{\rho, \varepsilon}\right)+J_{\rho}\left(u^{\rho}\right)}, \\
h^{\rho, \mathcal{\varepsilon}} & =\frac{2 E\left[\gamma\left(y_{0}^{\rho}\right)-\gamma\left(y_{0}\right)+\rho\right]}{J_{\rho}\left(u^{\rho, \varepsilon}\right)+J_{\rho}\left(u^{\rho}\right)} .
\end{aligned}
$$

Now, let $\left(p_{t}^{\rho, \mathcal{\varepsilon}}, q_{t}^{\rho, \varepsilon}, w_{t}^{\rho, \mathcal{\varepsilon}}, k_{t}^{\rho, \varepsilon}\right)$ be the solution of

$$
\left\{\begin{array}{l}
\left.-\mathrm{d} p_{t}^{\rho, \varepsilon}=\left[\left(b_{x}^{\rho}(t)\right)^{\tau} p_{t}^{\rho, \mathcal{\varepsilon}}-\left(f_{x}^{\rho}(t)\right)^{\tau} q_{t}^{\rho, \varepsilon}+\left(\sigma_{x}^{\rho}(t)\right)^{\tau} w_{t}^{\rho, \varepsilon}+\sum_{i=1}^{\infty}\left(g_{x}^{\rho}(t)\right)^{\tau} k_{t}^{\rho, \varepsilon}\right)^{i}\right] \mathrm{d} t, \\
-w_{t}^{\rho, \varepsilon} \mathrm{d} B_{t}-\sum_{i=1}^{\infty}\left(k_{t}^{\rho, \varepsilon}\right)^{i} \mathrm{~d} H_{t}^{i}, \\
\left.\mathrm{~d} q_{t}^{\rho, \varepsilon}=\left(f_{y}^{\rho}(t)\right)^{\tau} q_{t}^{\rho, \varepsilon} d t+\left(f_{z}^{\rho}(t)\right)^{\tau} q_{t}^{\rho, \varepsilon} d B_{t}+\sum_{i=1}^{\infty}\left(f_{r}^{\rho, i}(t)\right)^{\tau}\right) q_{t}^{\rho, \varepsilon} \mathrm{d} H_{t}^{i}, \\
q_{0}=-G_{0, y}\left(y_{0}^{\rho}\right) h_{0}^{\rho, \mathcal{\varepsilon}}+\gamma_{y}\left(y_{0}^{\rho}\right) h^{\rho, \varepsilon}, p_{T}=G_{1 x}\left(x_{T}^{\rho}\right) h_{1}^{\rho, \mathcal{\varepsilon}}-\Phi_{x}\left(x_{T}^{\rho}\right) q_{T}^{\rho, \mathcal{\varepsilon}}, \quad t \in[0, T] .
\end{array}\right.
$$


Here, $\quad b_{x}^{\rho}(t)=b_{x}\left(t, x_{t}^{\rho}, u_{t}^{\rho}\right), \quad \sigma_{x}^{\rho}(t)=\sigma_{x}\left(t, x_{t}^{\rho}\right)$, $f_{y}^{\rho}(t)=f_{y}\left(t, x_{t}^{\rho}, y_{t}^{\rho}, z_{t}^{\rho}, r_{t}^{\rho}, u_{t}^{\rho}\right)$, etc. Applying It $\widehat{o}$ 's formula to $\left\langle X_{t}^{\rho}, q_{t}^{\rho, \varepsilon}\right\rangle+\left\langle Y_{t}^{\rho}, p_{t}^{\rho, \varepsilon}\right\rangle$, variational inequality (59) can be rewritten as

$$
\begin{aligned}
& H\left(t, x_{t}^{\rho}, y_{t}^{\rho}, z_{t}^{\rho}, r_{t}^{\rho}, u_{t}^{\rho, \varepsilon}, p_{t}^{\rho, \varepsilon}, q_{t}^{\rho, \varepsilon}, w_{t}^{\rho, \varepsilon}, k_{t}^{\rho, \varepsilon}\right) \\
& \quad-H\left(t, x_{t}^{\rho}, y_{t}^{\rho}, z_{t}^{\rho}, r_{t}^{\rho}, u_{t}^{\rho}, p_{t}^{\rho, \varepsilon}, q_{t}^{\rho, \varepsilon}, w_{t}^{\rho, \varepsilon}, k_{t}^{\rho, \varepsilon}\right) \\
& \quad+\varepsilon \sqrt{\rho}+o(\varepsilon) \geq 0, \quad \forall v_{t} \in U, \text { a.e., a.s., }
\end{aligned}
$$

where the Hamiltonian function $H$ is defined as (44). Since

$$
\lim _{\varepsilon \longrightarrow 0}\left(\left|h_{0}^{\rho, \varepsilon}\right|^{2}+\left|h_{1}^{\rho, \varepsilon}\right|^{2}+\left|h^{\rho, \varepsilon}\right|^{2}\right)=1,
$$

there exists a convergent subsequence, still denoted by $\left(h_{0}^{\rho, \varepsilon}, h_{1}^{\rho, \varepsilon}, h^{\rho, \varepsilon}\right)$ such that $\left(h_{0}^{\rho, \varepsilon}, h_{1}^{\rho, \varepsilon}, h^{\rho, \varepsilon}\right) \longrightarrow\left(h_{0}^{\rho}, h_{1}^{\rho}, h^{\rho}\right)$ when $\varepsilon \longrightarrow 0$ with $\left|h_{0}^{\rho}\right|^{2}+\left|h_{1}^{\rho}\right|^{2}+\left|h^{\rho}\right|^{2}=1$.

Let $\left(p_{t}^{\rho}, q_{t}^{\rho}, w_{t}^{\rho}, k_{t}^{\rho}\right)$ be the solution of

$$
\left\{\begin{array}{l}
\left.-\mathrm{d} p_{t}^{\rho}=\left[\left(b_{x}^{\rho}(t)\right)^{\tau} p_{t}^{\rho}-\left(f_{x}^{\rho}(t)\right)^{\tau} q_{t}^{\rho}+\left(\sigma_{x}^{\rho}(t)\right)^{\tau} w_{t}^{\rho}+\sum_{i=1}^{\infty}\left(g_{x}^{\rho}(t)\right)^{\tau} k_{t}^{\rho}\right)^{i}\right] \mathrm{d} t, \\
-w_{t}^{\rho} \mathrm{d} B_{t}-\sum_{i=1}^{\infty}\left(k_{t}^{\rho}\right)^{i} \mathrm{~d} H_{t}^{i}, \\
\mathrm{~d} q_{t}^{\rho}=\left(\left(f_{y}^{\rho}(t)\right)^{\tau} q_{t}^{\rho} \mathrm{d} t+\left(f_{z}^{\rho}(t)\right)^{\tau} q_{t}^{\rho} \mathrm{d} B_{t}+\sum_{i=1}^{\infty}\left(f_{r}^{\rho, i}(t)\right)^{\tau}\right) q_{t}^{\rho} \mathrm{d} H_{t}^{i}, \\
q_{0}=-G_{0, y}\left(y_{0}^{\rho}\right) h_{0}^{\rho}+\gamma_{y}\left(y_{0}^{\rho}\right) h^{\rho}, p_{T}=G_{1 x}\left(x_{T}^{\rho}\right) h_{1}^{\rho}-\Phi_{x}\left(x_{T}^{\rho}\right) q_{T}^{\rho}, \quad t \in[0, T] .
\end{array}\right.
$$

From the continuous dependence of the solutions of FBSDEs with Lévy process on the parameters, we can prove that the following convergence holds: $\left(p_{t}^{\rho, \mathcal{\varepsilon}}, q_{t}^{\rho, \mathcal{\varepsilon}}, w_{t}^{\rho, \mathcal{\varepsilon}}, k_{t}^{\rho, \mathcal{\varepsilon}}\right) \longrightarrow\left(p_{t}^{\rho}, q_{t}^{\rho}, w_{t}^{\rho}, k_{t}^{\rho}\right)$; then, in equation (62), it implies that

$$
\begin{aligned}
& H\left(t, x_{t}^{\rho}, y_{t}^{\rho}, z_{t}^{\rho}, r_{t}^{\rho}, v_{t}, p_{t}^{\rho}, q_{t}^{\rho}, w_{t}^{\rho}, k_{t}^{\rho}\right) \\
& \quad-H\left(t, x_{t}^{\rho}, y_{t}^{\rho}, z_{t}^{\rho}, r_{t}^{\rho}, u_{t}^{\rho}, p_{t}^{\rho}, q_{t}^{\rho}, w_{t}^{\rho}, k_{t}^{\rho}\right) \\
& \quad+\sqrt{\rho} \geq 0, \quad \forall v_{t} \in U, \text { a.e., a.s.. }
\end{aligned}
$$

Similarly, there exists a convergent subsequence $\left(h_{0}^{\rho}, h_{1}^{\rho}, h^{\rho}\right)$ such that $\left(h_{0}^{\rho}, h_{1}^{\rho}, h^{\rho}\right) \longrightarrow\left(h_{0}, h_{1}, h\right)$ when $\rho \longrightarrow 0$ with $\left|h_{0}\right|^{2}+\left|h_{1}\right|^{2}+|h|^{2}=1$. Since $u_{t}^{\rho} \longrightarrow u_{t}^{*}$, as $\rho \longrightarrow 0$, we have $\left(x_{t}^{\rho}, y_{t}^{\rho}, z_{t}^{\rho}, r_{t}^{\rho}\right) \longrightarrow\left(x_{t}^{*}, y_{t}^{*}, z_{t}^{*}, r_{t}^{*}\right)$ and $\left(X_{t}^{\rho}, Y_{t}^{\rho}, Z_{t}^{\rho}, R_{t}^{\rho}\right) \longrightarrow\left(X_{t}, Y_{t}, Z_{t}, R_{t}\right)$, which is the solution of the variational equation as same as (6).

The following adjoint equation is introduced:

$$
\left\{\begin{array}{l}
\left.-\mathrm{d} p_{t}=\left[\left(b_{x}(t)\right)^{\tau} p_{t}-\left(f_{x}(t)\right)^{\tau} q_{t}+\left(\sigma_{x}(t)\right)^{\tau} w_{t}+\sum_{i=1}^{\infty}\left(g_{x}(t)\right)^{\tau} k_{t}\right)^{i}\right] \mathrm{d} t, \\
-w_{t} \mathrm{~d} B_{t}-\sum_{i=1}^{\infty}\left(k_{t}\right)^{i} \mathrm{~d} H_{t}^{i}, \\
\mathrm{~d} q_{t}=\left(\left(f_{y}(t)\right)^{\tau} q_{t} \mathrm{~d} t+\left(f_{z}(t)\right)^{\tau} q_{t} \mathrm{~d} B_{t}+\sum_{i=1}^{\infty}\left(f_{r}^{\rho, i}(t)\right)^{\tau}\right) q_{t} \mathrm{~d} H_{t}^{i}, \\
q_{0}=-G_{0, y}\left(y_{0}\right) h_{0}+\gamma_{y}\left(y_{0}\right) h, p_{T}=G_{1 x}\left(x_{T}\right) h_{1}-\Phi_{x}\left(x_{T}\right) q_{T}, \quad t \in[0, T] .
\end{array}\right.
$$

Similarly, it can be proved that equation (66); then, there exists nonzero constant $\left(p_{t}^{\rho}, q_{t}^{\rho}, w_{t}^{\rho}, k_{t}^{\rho}\right) \longrightarrow\left(p_{t}, q_{t}, w_{t}, k_{t}\right) ;$ then, inequation $(65)$ implies $\left(h_{1}, h_{0}, h\right) \in\left(R^{n_{1}} \times R^{m_{1}} \times R\right)$ with $\left|h_{0}\right|^{2}+\left|h_{1}\right|^{2}+|h|^{2}=1$

$$
\begin{aligned}
& H\left(t, x_{t}^{*}, y_{t}^{*}, z_{t}^{*}, r_{t}^{*}, v_{t}, p_{t}, q_{t}, w_{t}, k_{t}\right) \\
& \quad-H\left(t, x_{t}^{*}, y_{t}^{*}, z_{t}^{*}, r_{t}^{*}, u_{t}^{*}, p_{t}, q_{t}, w_{t}, k_{t}\right) \geq 0, \forall v_{t} \in U \text {, a.e., a.s.. }
\end{aligned}
$$

Then, we get the following theorem:

Theorem 2. Let Assumptions 1-3 hold; $u_{t}^{*}$ is an optimal control, and the corresponding optimal state trajectories are $\left(x_{t}^{*}, y_{t}^{*}, z_{t}^{*}, r_{t}^{*}\right)$, and $\left(p_{t}, q_{t}, w_{t}, k_{t}\right)$ is the solution of adjoint such that, for any admissible control $v_{t} \in U_{a d}[0, T]$, the maximum condition (67) holds.

This conclusion can be drawn from the above analysis directly.

\section{A Financial Example}

In this section, we will study the problem of optimal consumption rate selection in the financial market, which will naturally inspire our research to the forward-backward stochastic optimal control problem in Section 3. 
Assume the investor's asset process $x_{t}(t \geq 0)$ in the financial market is described by the following stochastic differential equation with the Teugels martingale:

$$
\left\{\begin{array}{l}
\mathrm{d} x_{t}=\left[\mu_{t} x_{t}-C_{t}\right] \mathrm{d} t+\sigma_{t} x_{t} \mathrm{~d} B_{t}+\sum_{i=1}^{\infty} g_{t}^{i} x_{t} \mathrm{~d} H_{t}^{i}, \\
x_{0}=W>0, t \in[0, T],
\end{array}\right.
$$

where $\mu_{t}$ and $\sigma_{t} \neq 0$ are the expected return and volatility of the value process $x_{t}$ at time $t$, respectively, and $C_{t}$ is the consumption rate process. Assume $\mu_{t}, \sigma_{t}, g_{t}$, and $C_{t}$ are all uniformly bounded $\mathscr{F}_{t}$-measurable random processes. The purpose of investors is to select the optimal consumption strategy $C_{t}^{*}$ at time $t \geq 0$, minimizing the following recursive utility:

$$
J(C(\cdot))=E\left[y_{0}\right],
$$

where $y_{t}$ is the following backward stochastic process:

$$
\left\{\begin{array}{l}
-d y_{t}=\left[L e^{-r t} \frac{C_{t}^{1-R}}{1-R}-r y_{t}\right] \mathrm{d} t-z_{t} \mathrm{~d} B_{t}-\sum_{i=1}^{\infty} r_{t}^{i} \mathrm{~d} H_{t}^{i}, \\
y_{T}=-x_{T}>0, \quad t \in[0, T],
\end{array}\right.
$$

with constant $L>0$, the discount factor $r>0$, and the Arrow-Pratt measure of risk aversion $R \in(0,1)$.

If the consumption process $C_{t}$ is regarded as a control variable, then combining (68) with (70), we encounter the following control system:

$$
\left\{\begin{array}{l}
\mathrm{d} x_{t}=\left[\mu_{t} x_{t}-C_{t}\right] \mathrm{d} t+\sigma_{t} x_{t} \mathrm{~d} B_{t}+\sum_{i=1}^{\infty} g_{t}^{i} x_{t} \mathrm{~d} H_{t}^{i}, \\
-\mathrm{d} y_{t}=\left[L e^{-r t} \frac{C_{t}^{1-R}}{1-R}-r y_{t}\right] \mathrm{d} t-z_{t} \mathrm{~d} B_{t}-\sum_{i=1}^{\infty} r_{t}^{i} \mathrm{~d} H_{t}^{i}, \\
x_{0}=W>0, y_{T}=-x_{T}>0, \quad t \in[0, T],
\end{array}\right.
$$

which is obviously a special case of stochastic control system (2) with $b(t, x, u)=\mu_{t} x_{t}-C_{t}, \sigma(t, x)=\sigma_{t} x_{t}, g(t, x)=$ $g_{t}^{i} x_{t}, \Phi_{x}\left(x_{T}\right)=-X_{T}$ and $f(t, x, y, z, r, u)=L e^{-r t}\left(C_{t}^{1-R /}\right.$ $(1-R))-r y_{t}$.

We can check that both Assumptions 1 and 2 are satisfied. Then, we can use our maximum principle (Theorem 1) to solve the above optimization problem. Let $C_{t}^{*}$ be an optimal consumption rate and $x_{t}^{*}, y_{t}^{*}$ be the corresponding wealth process and recursive utility process. In this case, the Hamiltonian function $H$ reduces to

$$
\begin{aligned}
& H\left(t, x_{t}^{*}, y_{t}^{*}, C_{t}, p_{t}^{*}, q_{t}^{*}, w_{t}^{*}, k_{t}^{*}\right)=\left\langle p_{t}^{*}, \mu_{t} x_{t}^{*}-C_{t}\right\rangle \\
& +\left\langle w_{t}^{*}, \sigma_{t} x_{t}^{*}\right\rangle+\left\langle k_{t}^{*}, g_{t}^{i} x_{t}^{*}\right\rangle-\left\langle q_{t}^{*}, L e^{-r t} \frac{C_{t}^{1-R}}{1-R}-r y_{t}^{*}\right\rangle,
\end{aligned}
$$

and $\left(p_{t}^{*}, q_{t}^{*}, w_{t}^{*}, k_{t}^{*}\right)$ is the solution of the following adjoint equation:

$$
\left\{\begin{array}{l}
\mathrm{d} q_{t}=r q_{t} \mathrm{~d} t \\
-\mathrm{d} p_{t}=\left[\mu_{t} p_{t}+\sigma_{t} w_{t}+\sum_{i=1}^{\infty}\left(k_{t}^{i}\right)^{\tau} g_{t}^{i}\right] \mathrm{d} t-w_{t} \mathrm{~d} B_{t}-\sum_{i=1}^{\infty} k_{t}^{i} \mathrm{~d} H_{t}^{i}, \\
q_{0}=1, p_{T}=q_{T}, \quad t \in[0, T] .
\end{array}\right.
$$

According to the maximum principle (47), we have

$$
p_{t}^{*}=-L e^{-r t}\left(C_{t}^{*}\right)^{-R} q_{t}^{*},
$$

and the optimal consumption rate

$$
C_{t}^{*}=\left(-\frac{e^{r t}}{L} \frac{p_{t}^{*}}{q_{t}^{*}}\right)^{-1 / R}
$$

By solving FBSDE (73), we get $q_{t}^{*}=e^{r t}, p_{t}^{*}=e^{r t} e^{\mu(T-t)}$, and $w_{t}^{*}=k_{t}^{*}=0$. Then, we get the optimal consumption rate of the investor which is

$$
C_{t}^{*}=\left(-\frac{e^{r T}}{L} e^{\mu(T-t)}\right)^{-1 / R}, \quad t \in[0, T]
$$

\section{Conclusions}

In this paper, a nonconvex control domain case of the forward-backward stochastic control driven by Lévy process is considered, and we obtain the global stochastic maximum principle for this stochastic control problem. And then, the problem of stochastic control with initial and final state constraints on the state variables is discussed, and a necessary condition about existence of the optimal control is also acquired. A financial example of optimal consumption is discussed to illustrate the application of the stochastic maximum principle.

\section{Data Availability}

No data were used to support this study.

\section{Conflicts of Interest}

The authors declare that they have no conflicts of interest.

\section{Acknowledgments}

This work was supported by the NNSF (11701335), Shandong NSF (ZR2019BG015), NSSF (17BGL058), Shandong Provincial Higher Education Science and Technology Plan Project (J18KA236), and Shandong Women's College Highlevel Research Project Cultivation Foundation (2018GSPGJ07).

\section{References}

[1] L. S. Pontryakin, V. G. Boltyanskti, R. V. Gamkrelidze, and E. F. Mischenko, The Mathematical Theory of Optimal Control Processes, John Wiley, New York, NY, USA, 1962.

[2] S. Peng, "A general stochastic maximum principle for optimal control problems," SIAM Journal on Control and Optimization, vol. 28, no. 4, pp. 966-979, 1990. 
[3] S. G. Peng, "Backward stochastic differrntial equations and application to optimal control," Applied Mathematics \& Optimization, vol. 27, no. 4, pp. 125-144, 1993.

[4] W. S. Xu, "Stochastic maximum principle for optimal control problem of forward and backward system," Journal of Australian Mathematical Sciences, vol. 37, pp. 249-259, 1998B.

[5] Z. Wu, "Maximum principle for optimal control problem of fully coupled forward-backward stochastic control system," System Science and Mathematical Sciences, vol. 11, no. 3, pp. 249-259, 1998.

[6] J. T. Shi and Z. Wu, "The maximum principle for fully coupled forward-backward stochastic control system," Acta Automatica Sinica, vol. 32, no. 2, pp. 161-169, 2006.

[7] R. Situ, "A maximum principle for optimal controls of stochastic systems with random jumps," in Proceedings of the National Conference on Control Theory and its Applications, Qingdao, China, 1991.

[8] J. Shi and Z. Wu, "Maximum principle for forward-backward stochastic control system with random jumps and applications to finance," Journal of Systems Science and Complexity, vol. 23, no. 2, pp. 219-231, 2010.

[9] J. T. Shi, "Global maximum principle for forward-backward stochastic control system with Poisson jumps," Asian Journal of Control, vol. 16, no. 1, pp. 1-11, 2014.

[10] M. Liu, X. Wang, and H. Huang, "Maximum principle for forward-backward control system driven by Itô-Lévy processes under initial-terminal constraints," Mathematical Problems in Engineering, vol. 2017, pp. 1-13, 2017.

[11] S. Tang, "The maximum principle for partially observed optimal control of stochastic differential equations," SIAM Journal on Control and Optimization, vol. 36, no. 5, pp. 1596-1617, 1998.

[12] Q. Meng, "A maximum principle for optimal control problem of fully coupled forward-backward stochastic systems with partial information," Science in China Series A: Mathematics, vol. 52, no. 7, pp. 1579-1588, 2009.

[13] B. Øksendal and A. Sulem, "Maximum principles for optimal control of forward backward stichastic differential equations with jumps," SIAM J Control Optim \enleadertwodots, vol. 48, pp. 2845-2976, 2009.

[14] G. Wang and $\mathrm{Z}$. Wu, "Kalman-Bucy filtering equations of forward and backward stochastic systems and applications to recursive optimal control problems," Journal of Mathematical Analysis and Applications, vol. 342, no. 2, pp. 1280-1296, 2008.

[15] H. Xiao, "The maximum principle for partially observed optimal control of forward-backward stochastic systems with random jumps," Journal of Systems Science and Complexity, vol. 24, no. 6, pp. 1083-1099, 2011.

[16] G. Wang, Z. Wu, and J. Xiong, "Maximum principles for forward-backward stochastic control systems with correlated state and observation noises," SIAM Journal on Control and Optimization, vol. 51, no. 1, pp. 491-524, 2013.

[17] G. Wang, C. Zhang, and W. Zhang, "Stochastic maximum principle for mean-field type optimal control under partial information," IEEE Transactions on Automatic Control, vol. 59, no. 2, pp. 522-528, 2014.

[18] S. Q. Zhang, J. Xiong, and X. D. Liu, "Stochastic maximum principle for partially observed forward backward stochastic differential equations with jumps and regime switching," Science China Information Sciences, vol. 61, no. 7, pp. 1-13, 2018.

[19] J. Xiong, S. Q. Zhang, S. Zhang, and Y. Zhuang, "A partially observed non-zero sum differential game of forward- backward stochastic differential equations and its application in finance," Mathematical Control \& Related Fields, vol. 9, no. 2, pp. 257-276, 2019.

[20] D. Nualart and W. Schoutens, "Chaotic and predictable representations for Lévy processes," Stochastic Processes and Their Applications, vol. 90, no. 1, pp. 109-122, 2000.

[21] D. Nualart and W. Schoutens, "Backward stochastic differential equations and Feynman-Kac formula for Levy processes, with applications in finance," Bernoulli, vol. 7, no. 5, pp. 761-776, 2001.

[22] Q. Meng and M. Tang, "Necessary and sufficient conditions for optimal control of stochastic systems associated with Lévy processes," Science in China Series F: Information Sciences, vol. 52, no. 11, pp. 1982-1992, 2009.

[23] M. Tang and Q. Zhang, "Optimal variational principle for backward stochastic control systems associated with Lévy processes," Science China Mathematics, vol. 55, no. 4, pp. 745-761, 2012.

[24] F. Baghery, N. Khelfallah, B. Mezerdi, and I. Turpin, "Fully coupled forward backward stochastic differential equations driven by Lévy processes and application to differential games," De Gruyter, vol. 22, no. 3, pp. 151-161, 2014.

[25] F. Zhang, M. N. Tang, and Q. X. Meng, "Sochastic maximum principle for forward-backward stochastic control systems associated with Lévy processes," Chinese Annals of Mathematics, vol. 35A, no. 1, pp. 83-100, 2014.

[26] X. R. Wang and H. Huang, "Maximum principle for forwardbackward stochastic control system driven by Lévy process," Mathematical Problems in Engineering, vol. 2015, Article ID 702802, 12 pages, 2015.

[27] H. Huang, X. Wang, and M. Liu, "A maximum principle for fully coupled forward-backward stochastic control system driven by Lévy process with terminal state constraints," Journal of Systems Science and Complexity, vol. 31, no. 4, pp. 859-874, 2018.

[28] P. Muthukumar and R. Deepa, "Infinite horizon optimal control of forward backward stochastic system driven by Teugels martingales with Lévy process," Stochastic and Dynamics, vol. 3, no. 17, pp. 1-17, 2017.

[29] J. B. Wu and Z. M. Liu, "Optimal control of mean-field backward doubly stochastic systems driven by Ito-Lévy processes," International Journal of Control, vol. 93, no. 4, pp. 953-970, 2018.

[30] W. Wang, J. Wu, and Z. Liu, "The optimal control of fullycoupled forward-backward doubly stochastic systems driven by Itô-Lévy processes," Journal of Systems Science and Complexity, vol. 32, no. 4, pp. 997-1018, 2019. 